\title{
BMJ Open Technology-enabled examinations of cardiac rhythm, optic nerve, oral health, tympanic membrane, gait and coordination evaluated jointly with routine health screenings: an observational study at the 2015 Kumbh Mela in India
}

Pratik Shah, ${ }^{1}$ Gregory Yauney, ${ }^{1}$ Otkrist Gupta, ${ }^{1}$ Vincent Patalano II, ${ }^{2,3}$ Mrinal Mohit, ${ }^{1}$ Rikin Merchant, ${ }^{4}$ S V Subramanian ${ }^{5}$

To cite: Shah P, Yauney G, Gupta 0, et al. Technologyenabled examinations of cardiac rhythm, optic nerve, oral health, tympanic membrane, gait and coordination evaluated jointly with routine health screenings: an observational study at the 2015 Kumbh Mela in India. BMJ Open 2018;8:e018774. doi:10.1136/ bmjopen-2017-018774

- Prepublication history and additional material for this paper are available online. To view these files, please visit the journal online (http://dx.doi. org/10.1136/bmjopen-2017018774).

PS and GY contributed equally.

Received 24 July 2017 Revised 12 February 2018 Accepted 22 February 2018

D) Check for updates

For numbered affiliations see end of article.

Correspondence to

Dr Pratik Shah; pratiks@mit.edu

\section{ABSTRACT}

Objectives Technology-enabled non-invasive diagnostic screening (TES) using smartphones and other point-ofcare medical devices was evaluated in conjunction with conventional routine health screenings for the primary care screening of patients.

Design Dental conditions, cardiac ECG arrhythmias, tympanic membrane disorders, blood oxygenation levels, optic nerve disorders and neurological fitness were evaluated using FDA-approved advanced smartphone powered technologies. Routine health screenings were also conducted. A novel remote web platform was developed to allow expert physicians to examine TES data and compare efficacy with routine health screenings. Setting The study was conducted at a primary care centre during the 2015 Kumbh Mela in Maharashtra, India. Participants 494 consenting 18-90 years old adults attending the 2015 Kumbh Mela were tested.

Results TES and routine health screenings identified unique clinical conditions in distinct patients. Intraoral fluorescent imaging classified $63.3 \%$ of the population with dental caries and periodontal diseases. An association between poor oral health and cardiovascular illnesses was also identified. Tympanic membrane imaging detected eardrum abnormalities in $13.0 \%$ of the population, several with a medical history of hearing difficulties. Gait and coordination issues were discovered in eight subjects and one subject had arrhythmia. Cross-correlations were observed between low oxygen saturation and low body mass index (BMI) with smokers $(p=0.0087$ and $p=0.0122$, respectively), and high BMI was associated with elevated blood pressure in middle-aged subjects.

Conclusions TES synergistically identified clinically significant abnormalities in several subjects who otherwise presented as normal in routine health screenings. Physicians validated TES findings and used routine health screening data and medical history responses for comprehensive diagnoses for at-risk patients. TES identified high prevalence of oral diseases, hypertension,
Strengths and limitations of this study

The strength of this work is that it is one of the first studies to investigate using technology-enabled mobile health screenings to augment routine health examinations.

- The study describes development and successful use of web examination platforms that enabled multiple physicians to diagnose health conditions remotely.

- A limitation of this study is that sample sizes for each test were different with respect to number of subjects and gender distribution.

- This study is limited to cross-sectional analysis. A future longitudinal study may allow for additional insights into time-varying conditions.

obesity and ophthalmic conditions among the middle-aged and elderly Indian population, calling for public health interventions.

\section{INTRODUCTION}

Providing good healthcare in low-income and middle-income countries (LMIC) paradoxically requires expensive equipment for health monitoring and assessment which may not be easily available because of resource limitations. ${ }^{1}$ Cardiovascular diseases, preventable blindness, oral cancer and treatable neurological conditions constitute more than half of the disease burden in LMIC and result in significant morbidity and mortality. ${ }^{2}{ }^{3}$ India, with a population in excess of 1.2 billion individuals, is one of the largest countries in the world. ${ }^{4}$ India has significant disparity in 
access to basic healthcare and diagnostic screenings due to its geographically fragmented medical infrastructure. ${ }^{5}$ Consequently, significant portions of the population may exist either as undiagnosed, diagnosed but unaware or misdiagnosed for several high-risk diseases at the primary care level.

Inexpensive device-based imaging and first-level analysis (eg, smartphones capable of pulse oximetry, blood pressure (BP), ECG recording and analysis or image segmentation) either operated by human experts, by operators with basic training using algorithms or clinical decision support systems are examples of affordable and potentially scalable technology-enabled screening (TES). ${ }^{6} 7$ Previous reports from our group have demonstrated the utility of smartphones, modular devices and imaging technologies for sleep apnoea ${ }^{8}$ and refractometry screenings, ${ }^{9}$ at-home monitoring of diabetic retinopathy ${ }^{10}$ and detecting melanomas. ${ }^{11}$ Using smartphones with low-cost adapters, other researchers have also performed oral and cervical cancer, ${ }^{12}$ diabetic retinopathy, ${ }^{13}$ and malaria ${ }^{14}$ assessments. Mobile smartphones equipped with imaging adapters, high-resolution cameras, light emitting diodes, fast processors and lightweight apps can thus be used for targeted diagnostic screenings at modest expense. ${ }^{1516}$

The majority of previous studies using newer TES approaches have been performed in silos concentrating on individual devices or specific anatomical sites, often precluding more comprehensive assessment of patient health. ${ }^{17-23}$ Interpretation of TES data has also been limited by risk of bias, differences between study groups and lack of comparison to establish routine health screenings that are otherwise commonly deployed in primary care screenings. Due to these reasons, a lack of consensus exists about the usefulness of TES in augmenting primary health screenings in LMIC. Therefore, there is a pressing need for cost-effective, reliable screening protocols and deployable technologies to empower LMIC medical professionals and healthcare providers to identify patients and add them to the continuum of care.

The 2015 Kumbh Mela mass gathering ${ }^{24}$ presented a unique opportunity for deployment and side-by-side evaluation of TES and routine health screenings. Multiple TES devices and methods and a remote clinical examination system to facilitate examination of findings were used to evaluate their collective use for comprehensive diagnoses of consenting adults. This study assesses whether low cost, portable and non-invasive examinations using TES can augment conventional routine health screenings by detecting additional anatomical, structural or biomarker-driven disease pathologies.

\section{METHODS}

\section{Study design}

Four hundred and ninety-four consenting adults between the ages of 18 and 90 years were screened by multiple tests in the order outlined in figure 1 at the Mahatma Gandhi Vidyamandir's Karmaveer Bhausaheb Hiray Dental College and Hospital (MGVKBHDC) in Nashik, India, during the 2015 Sinhast Kumbh Mela (14 July to 25 September). Table 1 shows the number of subjects who completed each test. Subjects could exit the study at their convenience, and the study design allowed for different numbers of subjects for each test (table 1). Inclusion criteria, ethical consideration and consent procedure are described in the (online supplementary appendix).

\section{Medical questionnaire and routine health screenings}

A designated physician administered a medical questionnaire, where subjects provided verbal answers and the physician was responsible for entering their answers into a computerised interface. The detailed questionnaire

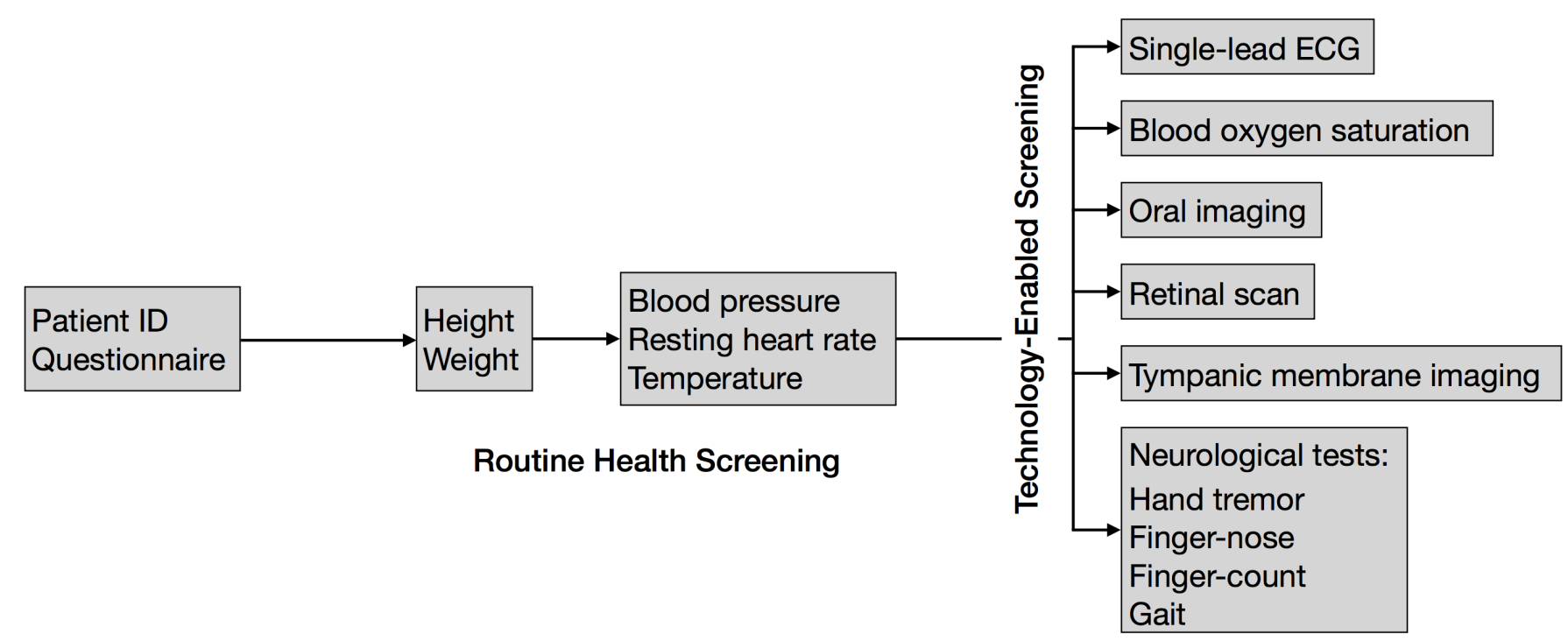

Figure 1 Study design. Flow chart for overall screening procedure. 
Table 1 Results of routine health screenings and technology-enabled screenings

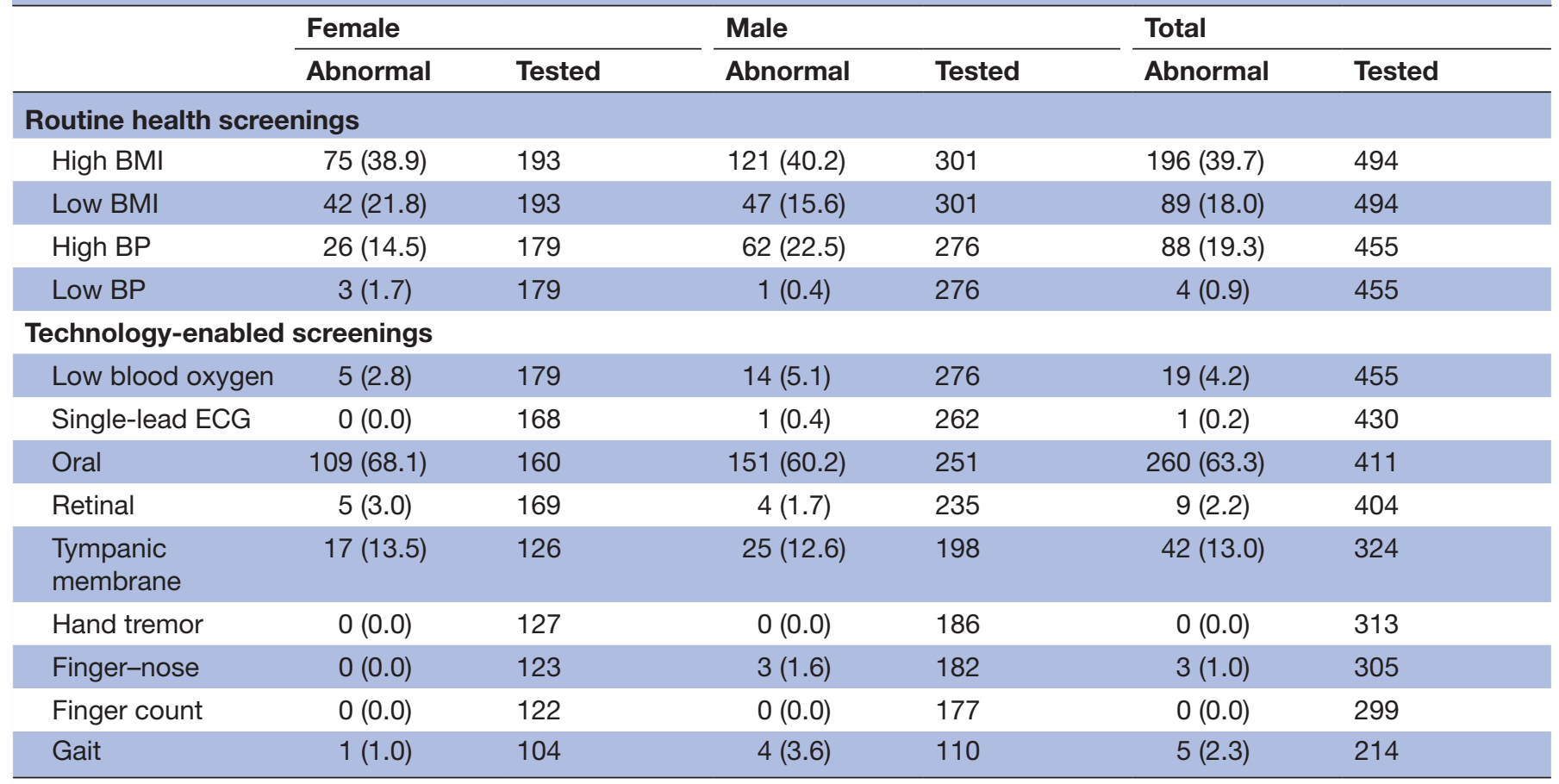

Numbers and percentages (in parentheses) of males and females with conditions identified by routine health screenings and technologyenabled screenings.

BMI, body mass index; BP, blood pressure.

included geographical and demographic questions as well as questions about medical history and current illnesses but did not capture data from past healthcare records. Height, weight, systolic and diastolic BP, resting heart rate, and temperature were also each measured, separately by a different physician.

\section{Technology-enabled screening}

Food and Drug Administration (FDA) -approved devices were used to image patients, CellScope Oto (CellScope, USA) for the tympanic membrane, D-EYE Direct Ophthalmoscopy Adapter (D-EYE Srl, USA) for the optic nerve head and SOPROCARE (SOPRO Acteon Imaging, France) for dental health. Microsoft Kinect (Microsoft, USA) was used to record subjects performing gait and coordination tests (online supplementary figure 1). AliveCor Mobile ECG (AliveCor, USA) was used to capture and analyse a $30 \mathrm{~s}$ rhythm strip. A CMS 50 DL Pulse Oximeter (Contec Medical Systems, USA) was used to measure blood oxygen saturation levels of haemoglobin. All devices and clinical evaluations have been previously described elsewhere. ${ }^{17-20} 23$ 25-29 The (online supplementary appendix) details specific procedures for each device and the principles by which each operates.

\section{Data analyses}

Expert physicians conducted diagnostic feature annotation of de-identified images and videos collected by TES via a web-based examination portal in order to maximise time in the field for screening additional subjects (online supplementary methods). This password-protected secure interface displayed an image or video for one patient at a time for a given examination. Physicians were able to mark specific features in the videos by drawing boxes around them that paused that specific frame, assign an overall score of 1 (best) to 5 (worst) for the entire video, and to write clinical features that were present for specific frames or the entire video (online supplementary figure 2). A panel of at least three physicians for each type of examination was assembled who remotely and independently annotated the data facilitated by the web interface. The majority ratings for each subject were then calculated for all TES tests. For subjects with no majority rating, the lesser of the tied ratings was chosen to not overstate the prevalence of diagnosed illnesses. On average, each physician spent tens of seconds to approximately a minute annotating each video. Results from each test were analysed for cross-correlations with medical questionnaire responses, age and sex. Analyses of results from specific tests include data from all subjects who completed that test.

\section{RESULTS}

\section{Medical history and routine health screenings}

Results of routine health screenings are shown in table 1, with gender and age range breakdowns in (online supplementary table 1$)$. Medical questionnaire responses are shown in (online supplementary tables 2-4). Accepted clinical ranges for each condition identified by routine health screenings were specified in consultation with physicians and were applied automatically to the routine 
health screening data without requiring physicians to annotate the data on a per-patient basis (online supplementary methods)..$^{25} 30$ Obesity $(39.7 \%)$ and elevated BP $(19.3 \%)$ were identified as most prevalent among the screened population. In comparing our data to the latest release of the National Family and Health Survey, we detected higher prevalence of high body mass index (BMI) and elevated BP for both women and men but a similar prevalence of low BMI (online supplementary tables 5-6). ${ }^{32}$

\section{Population demographic analysis}

It took an average of approximately $35 \mathrm{~min}$ for each patient to complete the medical questionnaire, routine health screenings and TES screening. Overall, there were more male than female participants which is likely at least in part the consequence of the fact that there are generally more male pilgrims at outdoor Indian religious festivals, including the Kumbh Mela. The gender breakdown for nearly all tests was approximately $60 \%$ males and $40 \%$ females, though gait analysis had an equal per cent of both because the subjects who stayed long enough to complete that screening happened by chance to be split equally among males and females (online supplementary figure 3). Adolescents (18 and 19 years of age) and old adults (65-90 years of age) were approximately $30 \%$ of the total population (online supplementary figure 4). The remaining $70 \%$ of the population comprised approximately $31 \%$ of young adults (20-39 years) and approximately $39 \%$ middle age (40-64 years) subjects (online supplementary figure 4 ). Threefold more female than male adolescents and fourfold more old-aged males than old-aged females participated in the study, whereas other age groups had roughly equal numbers of males and females (online supplementary table 1).

\section{Medical questionnaire responses}

Medical histories of dental issues, swollen joints, hearing difficulties and leg cramps were each reported by $26.1 \%$, $25.3 \%, 21.9 \%, 18.6 \%$ of the population, respectively (online supplementary table 2). Several respondents reported a history of diabetes, BP and cardiovascular diseases in their families. $8.9 \%$ of total respondents reported that they had been diagnosed with high BP, and $6.3 \%$ were being treated for the disease (online supplementary table 2 ). Subjects diagnosed with a certain clinical condition and/or undergoing treatment for it may not be the same individuals. Fifty per cent out of 494 respondents reported they wear glasses, indicating that they have refractive error vision problems (online supplementary table 2). Roughly equal numbers of both sexes responded yes to the majority of questions, with the exception of tobacco addiction, which was reported almost exclusively by males (online supplementary table 2). Online supplementary table 3 shows the age-cohort distribution of percentage of the people who said yes to a particular question (eg, approximately $17 \%$ of the 98 subjects who said they had family history of diabetes were 18-19year olds). Barring family history of diabetes, high BP, and thyroid and cardiovascular diseases; higher percentages of middle-aged and older-aged adults said yes to the majority of all other questions (online supplementary table 3). Additionally, higher percentages of individuals in the middle and old age groups versus adolescents and young adults answered yes to medical history questions (eg, approximately $35 \%$ of the 65 subjects in the 18-19 age group said they had a vision problem vs approximately $67 \%$ and approximately $75 \%$ of $40-64$ and 65-90year olds) (online supplementary table 4). See (online supplementary tables 2-4), each shows the full list of questions asked in the medical questionnaire.

\section{Prevalence of obesity and hypertension in males and low BMI in young females}

Routine health screenings showed that approximately $19.3 \%$ of 455 subjects in the study had elevated BP, and $0.9 \%$ had lower than normal BP (table 1). $22.5 \%$ of the 276 tested men of all age groups had elevated BP measurements compared with $14.5 \%$ of the 179 tested women (Table 1). High BMI was measured in $39.7 \%$ of the tested population (table 1). Middle age adults (40-64 years) had statistically significantly higher BMI than young adults (20-39 years) and old age subjects (65-90 years) (online supplementary table 7). Middle-aged and old-age subjects also had statistically significantly higher BP than adolescents and adult participants in the study (online supplementary table 7). Approximately, $18.0 \%$ of the tested population was underweight (Table 1). More women in the 18-19 and 20-39 age groups and more men in the 40-64 and 65-90 age groups had lower BMI than subjects in other age groups (online supplementary table 5). Overall, more men aged 20-39 were found to be obese compared with women of that age group $(\mathrm{p}=0.0375)$ who in fact were scored as underweight by statistical significance. Forty-two subjects, the majority in the 40-64 age group, suffered from hypertension and had high BMI. Underweight men and women between the ages of 40 and 64 also found to be at higher risk for elevated BP $(n=9)$.

Supplementary data

\section{Comparisons between medical questionnaire and results from} routine health screenings

Adults aged 40 years and older of both sexes who were classified as abnormal in any routine health screening were the largest group of subjects correspondingly reporting family history, being diagnosed with or receiving treatment for several diseases on the questionnaire. Online supplementary table 8 lists $\mathrm{p}$ values and percentages for cross-correlations between medical histories and results from routine health screenings. Statistically significant correlations between obesity and subjects who wore glasses $(58.7 \%)$, reported high BP $(13.3 \%)$ or had a family history of diabetes $(24.5 \%)$, or hypertension $(20.9 \%)$ were identified $(\mathrm{p}=0.0024, \mathrm{p}=0.0091, \mathrm{p}=0.0384$, 

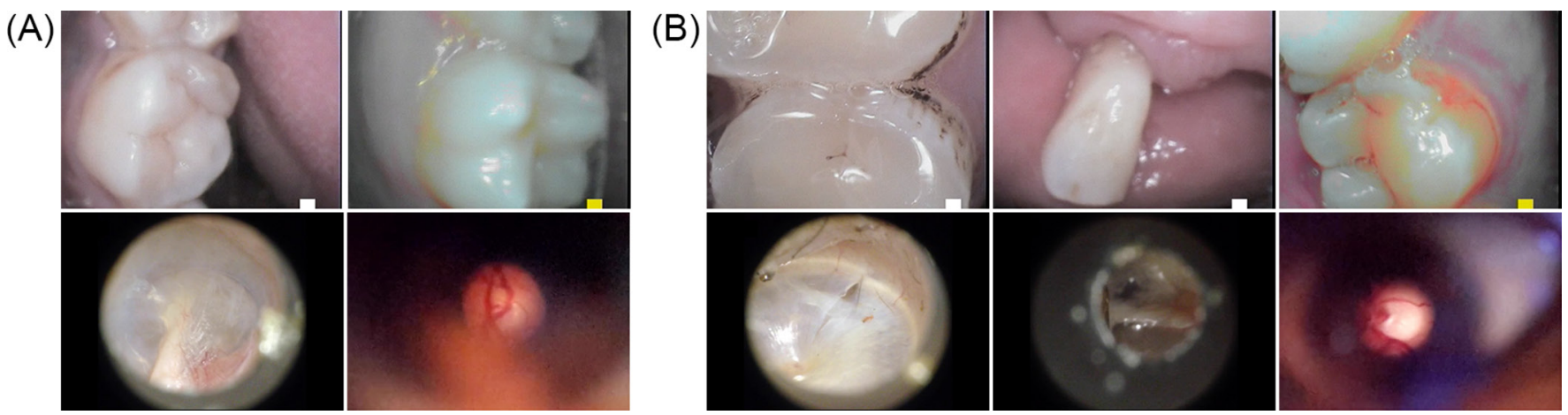

Figure 2 Representative images of labelled conditions detected in technology-enabled screenings (TES). (A) Normal images for TES. Left to right, top to bottom: dental, periodontal, tympanic membrane, optic nerve. (B) Labelled conditions for TES. Left to right, top to bottom: caries, missing teeth, periodontal disease, perforated eardrum, effusion, width of optic rim 0.01-0.1.

$\mathrm{p}=0.0173$, respectively) (online supplementary table 8 ). Interestingly, overweight subjects were more statistically significantly likely to be non-smokers; conversely, underweight subjects were more likely to be either addicted to tobacco and/or smokers ( $\mathrm{p}=0.0406, \mathrm{p}=0.0122$, respectively). Occurrences of high BP cross-correlated with several groups of individuals' with a medical history of swollen joints, difficulty walking and diabetes $(\mathrm{p}=0.0425$, $\mathrm{p}=0.0483, \mathrm{p}=0.0004$, respectively). Subjects reporting either history of hypertension $(\mathrm{n}=7)$ or undergoing hypertension treatment $(\mathrm{n}=14)$ were statistically significantly more likely $(\mathrm{p}=0.0006, \mathrm{p}=0.0002)$ to be measured with high BP during our screening (online supplementary table 8 ). High BMI and high BP were additionally found to be correlated with many conditions on the medical questionnaire (online supplementary table 9).

\section{Identification of clinical conditions using TES}

Figure 2 shows representative diagnostic images and associated diagnoses captured using TES. Intraoral fluorescent $(63.3 \%)$, tympanic membrane $(13.0 \%)$ and oxygen saturation $(4.2 \%)$ imaging examinations identified the largest percentage of unhealthy subjects (table 1 ). Online supplementary table 10 shows the breakdown of abnormal results by gender and age range. Approximately, $38.0 \%$ of subjects had dental caries and $28.0 \%$ had one or more teeth missing. Periodontal diseases were found in $14.8 \%$ of the population (online supplementary table 11). Previously undiagnosed subjects with abnormalities in their ECG rhythm strip $(n=1)$, optic nerve imaging $(n=9)$, gait analysis $(n=5)$ and finger-nose coordination $(n=3)$ test results were identified (table 1 ). The expert physicians annotated all nine subjects identified with abnormal optic nerve heads with a cup-to-disc ratio more than 0.3. All subjects who completed the hand tremor and fingercount tests were found to be normal.

A slightly larger percentage of tested female participants compared with males were determined to be unhealthy across the majority of TES tests, although the differences were not statistically significant with the exception of middle-aged females who were statistically more likely to have poor dental health than middleaged males $(\mathrm{p}=0.0266)$. Middle-aged $(\mathrm{n}=120)$ and older $(\mathrm{n}=66)$ adults of both sexes encompassed $70.7 \%$ of all subjects with abnormal oral TES results (online supplementary table 10). Statistically higher prevalence of dental diseases was measured in 65-90year olds and 40-64year olds compared with 18-19year olds and $20-39$ year olds $(\mathrm{p}<0.0001$ in all four cases). Low blood oxygen saturation and abnormal tympanic membranes were found in 13 and 26 subjects, respectively, who also had dental issues (online supplementary table 12). One subject each had low blood oxygen, tympanic membrane problems and unsatisfactory performance in the gait test. Adults aged 40 and above of both sexes were the majority of the subjects who failed two TES tests.

\section{Identification of subjects with abnormal routine health screenings and TES results}

Numbers of subjects who were scored not normal in one test each from TES and routine health screening are shown in (online supplementary table 13). High and low BMI followed by elevated BP measurements were most prevalent in subjects with abnormal TES results. Underweight individuals made up $18.0 \%$ of the population (table 1), and $42.1 \%$ of these individuals also had low blood oxygen saturation $(\mathrm{p}=0.0110)$ (online supplementary table 13). Similarly, approximately one-third of the individuals with tympanic membrane abnormalities also had low BMI. Abnormal optic disc diameters were measured in four overweight participants (online supplementary table 13). Dental screening identified the largest numbers of unhealthy subjects from the population who were measured with either high BMI, low BMI or elevated $\mathrm{BP}$, but these cross-correlations were not statistically significant due to extensive prevalence poor oral health in the community. Overall, high BMI $(39.7 \%)$ and elevated BP $(19.3 \%)$ and poor dental health $(63.3 \%)$ were widespread in middle-aged and older adults of the population (table 1, online supplementary tables 5 and 10 ).

\section{Comparisons between medical questionnaire and results from} TES

Table 2 shows percentages of subjects who were scored as not normal in a particular TES test and responded yes to a medical history question. Individuals with swollen joints, 
Table 2 Number of subjects identified with a clinical condition by a technology-enabled screening test and responded yes to a medical history question

\begin{tabular}{|c|c|c|c|c|c|c|}
\hline & Hypoxaemia (19) & TM (42) & Retinal (9) & Oral (260) & Finger-nose (3) & Gait (5) \\
\hline Glasses & 7 & 22 & 6 & $145^{*}$ & 1 & 1 \\
\hline Dental & 9 & 14 & 5 & $82^{*}$ & 1 & $4^{*}$ \\
\hline Swollen joints & 3 & 14 & 4 & $85^{*}$ & $3^{*}$ & $4^{*}$ \\
\hline Hearing & 5 & 17 & 4 & $72^{*}$ & 2 & 3 \\
\hline FH diabetes & 2 & 7 & 2 & 41 & 0 & 1 \\
\hline FH high BP & 0 & 6 & 2 & $31^{\star *}$ & 0 & 0 \\
\hline Tobacco & 3 & 3 & 0 & 27 & 1 & 0 \\
\hline Difficulty walking & 3 & 5 & $3^{*}$ & $36^{\star}$ & $3^{\star}$ & 1 \\
\hline High BP & 0 & 5 & 1 & 30 & 0 & 0 \\
\hline Diabetes & 0 & 4 & 0 & 23 & 0 & 0 \\
\hline High BP Rx & 0 & 4 & 1 & $24^{*}$ & 0 & 0 \\
\hline Asthma & 3 & 3 & 0 & 15 & 1 & 0 \\
\hline Smoking & $4^{*}$ & 1 & 0 & 13 & 0 & 1 \\
\hline FH cardiac & 0 & 2 & 0 & 7 & 0 & 0 \\
\hline Cardiac Rx & 1 & 1 & 1 & $8^{*}$ & 0 & 0 \\
\hline Cardiovascular & 0 & 1 & 1 & 4 & 0 & 0 \\
\hline Low BP & 0 & 0 & 0 & 3 & 0 & 0 \\
\hline FH stroke & 0 & 1 & 0 & 2 & 0 & 0 \\
\hline FH eye disease & 0 & 0 & 0 & 3 & 0 & 0 \\
\hline Heart attack & 0 & 0 & 0 & 3 & 0 & 0 \\
\hline Coronary bypass & 0 & 0 & 0 & 3 & 0 & 0 \\
\hline Drinking & 0 & 0 & 0 & 1 & 0 & 0 \\
\hline Eye treatment & 0 & 0 & 0 & 2 & 0 & 0 \\
\hline Memory loss & 0 & 0 & 0 & 2 & 0 & 0 \\
\hline Ear treatment & 0 & 1 & 0 & 1 & 0 & 0 \\
\hline $\mathrm{FH}$ ear disease & 0 & 0 & 0 & 1 & 0 & 0 \\
\hline
\end{tabular}

Total populations, in parentheses, reflect the number of subjects with the particular condition in that column identified by technology-enabled screenings. Multiple subjects were associated with more than one condition or questionnaire response.

${ }^{*} \mathrm{P}<0.05$, subjects are more likely to have responded yes to the column's question and have the condition.

${ }^{* *} \mathrm{P}<0.05$, subjects are less likely to have responded yes to the column's question and have the condition.

$\mathrm{BP}$, blood pressure; $\mathrm{FH}$, family history; TM, tympanic membrane; $\mathrm{Rx}$, prescription;

hearing and walking issues were found to be more likely to be scored as abnormal in TES tests compared with other groups. Four subjects $(21.1 \%)$ measured with low oxygen saturation had a medical history of being smokers and this correlation was statistically significant (online supplementary table 14). $40.5 \%$ of the subjects for whom we identified tympanic membrane abnormalities reported hearing issues $(p=0.0053)$, see (online supplementary table 14). Presence of dental caries, gingivitis and/or periodontal diseases was correlated with more statistically significant incidences of various clinical conditions reported in the medical questionnaire. High percentages of subjects scored as not normal in the finger-nose $(n=3)$ and or gait tests $(n=5)$ had reported hearing issues (table 2). Similar to results in routine health screenings, individuals aged 40 years and older of both sexes were the largest group of subjects who gave affirmative responses to the questionnaire and abnormal TES results, although this was not statistically significant.

TES synergistically identifies unique subset of abnormal individuals in conjunction with routine health screenings Data from subjects who completed the medical questionnaire, all routine health screenings and all TES tests $(n=111)$ allowed comprehensive analyses. Subjects who exited the study before completing all routine health screenings and TES tests were not considered in these analyses. Routine health screenings identified 32 as normal and 79 as abnormal from these 111 subjects, compared with 41 normal and 70 abnormal subjects classified by TES (online supplementary table 15). Online supplementary tables 16-17 show the data for each of the 111 subjects. Our data indicate that a similar percentage of these 111 subjects benefited from diagnosis offered 
Table 3 Synergistic role of technology-enabled screening (TES) in identifying at-risk or sick individuals

\begin{tabular}{|c|c|c|c|c|c|c|c|c|c|c|}
\hline \multirow[b]{2}{*}{ RHS } & \multirow[b]{2}{*}{ TES } & \multicolumn{2}{|c|}{ Adolescent (18-19) } & \multicolumn{2}{|c|}{ Young adult (20-39) } & \multicolumn{2}{|c|}{ Middle age (40-64) } & \multicolumn{2}{|c|}{ Old age (65-90) } & \multirow[b]{2}{*}{ Total $(n=111)$} \\
\hline & & Female & Male & Female & Male & Female & Male & Female & Male & \\
\hline$\checkmark$ & $\checkmark$ & 5 & 0 & 3 & 0 & 0 & 2 & 0 & 0 & 10 \\
\hline$\checkmark$ & $x$ & 4 & 0 & 5 & 4 & 5 & 3 & 0 & 1 & 22 \\
\hline$x$ & $x$ & 5 & 0 & 3 & 7 & 9 & 15 & 2 & 7 & 48 \\
\hline
\end{tabular}

Check marks indicate normal status while $\mathrm{x}$ indicates abnormalities in a particular screening method.

$\mathrm{RHS}$, routine health screenings; TES, technology-enabled screening.

by TES as those screened by routine health screenings. Table 3 shows the age and gender profiles for 111 subjects and the differential diagnoses between the two screening methods.

Overall, we found abnormal BMI measurements and poor dentition led to the majority of the abnormalities in these 111. Tympanic membrane and BP abnormalities were the second-most widespread in this cohort. Medical questionnaire responses and TES screening results from these 111 subjects illustrate another use case with value for augmented screening. Several individuals with healthy medical histories were found to have either abnormal routine health screenings $(n=12)$ or TES results $(n=7)$ or both $(n=6)$. Dental and ear issues were the most common TES abnormalities associated with these subjects with healthy medical histories. Importantly, all seven subjects with a healthy medical questionnaire identified as abnormal by TES (dental, $n=5$; optic nerve, $n=1$; tympanic membrane, $n=1$ ) had no previous diagnoses of these conditions and were different from the 12 individuals with abnormal routine health screenings. These results indicate the unique and synergistic value of TES in providing comprehensive care in conjunction with routine health screenings.

\section{DISCUSSION}

Many of the subjects identified by TES did not have any abnormal routine health screenings, indicating that TES can play a role in identifying subjects who need care but would not be identified by routine health screenings alone. Each TES test identified distinctive abnormalities in different patients and played a distinct role in identifying at-risk or sick individuals. Because the TES tests screen for much different conditions than the routine health screenings, our results indicate mutually independent but not entirely mutually exclusive performances of both in identifying at-risk or sick subjects. We examined several potential moderators and old age was a statistically significant premonition for abnormal TES results, underscoring the crucial role for augmented screenings in middle-aged and geriatric individuals. Large proportions of subjects identified with abnormalities in oral $(69.1 \%)$, tympanic membrane $(59.5 \%)$ and retinal $(33.3 \%)$ tests, as well as the only subject in the single-lead ECG test, did not report their respective conditions on their medical questionnaires. TES, thus, facilitates more thorough and non-invasive primary care screenings and may expedite early interventions for conditions not identified by routine health screenings.

Routine health screenings provided valuable insights into the rising epidemics of hypertension and obesity in the screened population in our study. Approximately, $19.3 \%$ of the screened population in our study was suffering from hypertension. Eighteen per cent of the screened population in our study was underweight $(\mathrm{BMI}<18.5)$ and several of these subjects were hypoxic, with low oxygen saturation, which can be considered a proxy measure for anaemia. Pulse oximetry screenings identified 19 subjects with $<90 \%$ SpO2. Vold et al, in studies carried out in Norway, have reported high BMI, middle age and smoking as predictors of oxygen saturation. ${ }^{33}$ Subjects with low blood oxygen had medical histories of smoking addiction and asthma. Most of them were middle-aged men, and the majorities were underweight. We also detected optic nerve abnormalities, dental diseases, gait and hearing difficulties in subjects with low blood oxygen, suggesting that a poor overall health status may coexist with low blood oxygen measurements. Our data suggest the usefulness of deploying pulse oximetry for general health screenings, especially for underweight patients in LMIC.

Inadequate dental hygiene and resulting sequelae can have significant impact on quality of life and increase the risk of cardiovascular diseases. ${ }^{34} 35$ 'Remote monitoring' and 'Store-forward' approaches for dental examinations, reviewed elsewhere, ${ }^{36}$ have been used for teledentistry. In patients aged 65-74 years, the prevalence of caries was approximately $70 \%$ and multicentric oral health survey reported the prevalence of carries to be $51 \%-95 \% .{ }^{37} \mathrm{We}$ found approximately $48 \%$ of people aged 65-90years diagnosed with caries or periodontal disease. A high percentage of subjects aged 45-60 years in our study, who usually may not have been detected by traditional methods, were identified with poor dental health and may have been detected due to the more comprehensive evaluation offered by TES. Significant cross-correlations 
between patients reporting cardiovascular treatment with poor oral health $(\mathrm{p}=0.0267)$ was identified, underscoring the importance of routine dental care and calling for urgent attention to the oral disease epidemic in India.

There are approximately 11.2 million persons aged 40 years and older with glaucoma in India. ${ }^{38}$ The most common optic nerve abnormalities that were detected in our study were optic nerve cupping as a risk factor for glaucoma, and optic nerve head neovascularisation that is a sign of diabetic retinopathy. All nine patients who were scored as abnormal by optic nerve photography in our study had a Disc Damage Likelihood Scale scores of 3 , indicating early onset of glaucoma. The majority of these patients were females aged between 20 and 64 years whereas two old age males were marked abnormal. Four patients were also obese and high $\mathrm{BP}$ was measured in two individuals, highlighting vulnerable status of young and middle-aged obese Indian population.

Smartphone-enabled tele-otology has been deemed suitable for both on-site and remote diagnoses of tympanic membrane and Otitis media $(\mathrm{OM})$ previously. ${ }^{39}$ Perforated tympanic membranes $(59.2 \%)$ and/or effusions $(47.6 \%)$ were most prevalent in patients labelled as abnormal by Ear, Nose and Throat surgeons. These patients may have been suffering from OM infection and or a generalised inflammation. A large segment of these subjects were middle age or older adults and were also the predominant group that failed the gait and coordination test. Physician consensus, imaging data and a history of hearing issues emphasise the substantial challenges in diagnosing and treating these patients. We did not perform audiology or speeches tests with these subjects, and thus are unable to cross-correlate the imaging data with functional tests.

Due to acute shortages of trained neurologists, diagnoses of neurological disorders are extremely challenging in LMIC. ${ }^{40}$ Three subjects in the finger-nose test were identified with incoordination and dysmetria. Seven out of the eight total subjects failing neurological tests were males older than 40 years, and three were underweight. The majority of subjects failing either the gait and/or finger-nose tests a medical history of swollen joints and a few said that they had difficulty in walking (all subjects were able to walk unassisted). It is conceivable that these issues may be unlinked or causal to their performance in the gait test. However, none of these conditions had previously been diagnosed in these individuals reaffirming a need for more aggressive and large-scale screening using TES methods. We are working on generative algorithms for classification of various neurological disorders and hope to incorporate these in future studies.

Physicians using TES devices in our study commented that a brief period of acclimatisation was necessary in both use and interpretation of results from the devices before use in the study. Incorporating training for use of mobile technologies for diagnoses during medical school can reduce this learning curve. To our surprise, most of our tele-examination physicians could accurately diagnose presented conditions often with overwhelming consensus between them. Patients enrolled in our study were also pleased to see immediate results from TES and get rapid feedback from physicians. We acknowledge that TES devices may provide only an indication of the clinical pathologies they evaluate instead of a thorough diagnosis. Despite the claims made by device manufacturers, we also recognise that in their current form-factor TES will not replace or completely remove the physician from the loop. We used representative, clinically validated devices in this study and our diagnoses may be generalisable to findings from similar datasets. Due to some subjects leaving the study early, a number of patients examined by each TES test and routine health screening were not identical, restricting more comprehensive analyses to the 111 subjects who completed all tests. Although this work is one of the largest, if not the largest, studies carried out using a wide array of different TES methods, a larger sample size in follow-up studies may result in more comprehensive identification of patient demographics and the full gamut of clinical conditions. We do, however, emphasise that one goal of this study was to conduct a cross-sectional analysis of both the population and the tests instead of focusing on only one TES method with a large sample size.

While routine health screenings continue to be important, this study demonstrates that the emerging techniques of TES can play an important synergistic role in stratifying populations and providing personalised screening and care, especially in LMIC. Multiple TES screening methods and data analyses outlined in this study can help in training and standardisation for deployment of augmented, low cost, non-invasive and portable screening approaches in conjunction with traditional primary healthcare examinations, leading to increased clinical interventions, diagnoses and awareness of health conditions for individuals.

\section{Author affiliations}

${ }^{1}$ Media Lab, Massachusetts Institute of Technology, Cambridge, Massachusetts, USA ${ }^{2}$ Department of Ophthalmology, Cambridge Health Alliance, Cambridge,

Massachusetts, USA

${ }^{3}$ Department of Opthalmology, Harvard Medical School, Boston, Massachusetts, USA ${ }^{4}$ Department of Prosthodontics, Karmaveer Bhausaheb Hiray Dental College and Hospital, Nashik, Maharashtra, India

${ }^{5}$ Department of Social and Behavioral Sciences, Harvard T.H. Chan School of Public Health, Boston, Massachusetts, USA

Acknowledgements We thank physicians, staff, student volunteers and Dr Sanjay Bhawsar of MGVKBHDC for support for conducting this study, Dr Arun Jamkar and Maharashtra Health and Sciences University, India for guidance to select the location and Krishna Rastogi, Sathya Sai, Hisham Bedri, Geetanjali Rathore, Mayank Kumar and Akshat Wahi for technical assistance. Dr Judy-Fine Edelstein, Dr Diana Green and Tek Yadav of Cambridge Health Alliance, Cambridge, MA; Dr Maneesh Bapaye, Sunil Ugale, Dr Shirish Ghan and Dr Roma Bagi Nashik, India and Dr Tulio Valdez, Massachusetts Institute of Technology for help in expert clinical evaluations.

Contributors $\mathrm{OG}, \mathrm{MM}$ and GY organised the data received from collaborating institution by pooling various test data for each patient, $O G$ created a web analysis platform for remote examinations; PS, OG, SVS, VP and GY analysed and interpreted data labelled by expert physicians; PS, GY and OG performed literature search; GY made figures; PS and GY wrote the manuscript; RM led clinical data collection and 
coordinated transfer to MIT; PS supervised the research and directed the study at MIT.

Funding Massachusetts Institute of Technology Media Lab and Karmaveer Bhausaheb Hiray Dental College and Hospital departmental funds.

Competing interests None declared.

Patient consent Obtained.

Ethics approval The Mahatma Gandhi Vidyamandir's Karmaveer Bhausaheb Hiray Dental College and Hospital Institutional Ethics Committee reviewed and approved protocol MGVKBHDC/15-16/571 for clinical data collection in Nashik, India. Deidentified data were transferred and analysed at the Massachusetts Institute of Technology (MIT) in Cambridge, Massachusetts, according to MIT Committee on the Use of Humans as Experimental Subjects approval for protocol 1512338971.

Provenance and peer review Not commissioned; externally peer reviewed.

Data sharing statement Anonymised patient-level data and or full dataset will be made available following standard MIT Committee on the Use of Humans as Experimental Subjects data sharing protocols.

Open Access This is an Open Access article distributed in accordance with the Creative Commons Attribution Non Commercial (CC BY-NC 4.0) license, which permits others to distribute, remix, adapt, build upon this work non-commercially, and license their derivative works on different terms, provided the original work is properly cited and the use is non-commercial. See: http://creativecommons.org/ licenses/by-nc/4.0/

(c) Article author(s) (or their employer(s) unless otherwise stated in the text of the article) 2018. All rights reserved. No commercial use is permitted unless otherwise expressly granted.

\section{REFERENCES}

1. Rivo M. 2008 World Health Report emphasizes importance of primary health care. Am Fam Physician 2009;79:1051.

2. Alwan A, Maclean DR, Riley LM, et al. Monitoring and surveillance of chronic non-communicable diseases: progress and capacity in highburden countries. Lancet 2010;376:1861-8.

3. Samb B, Desai N, Nishtar S, et al. Prevention and management of chronic disease: a litmus test for health-systems strengthening in low-income and middle-income countries. Lancet 2010;376:1785-97.

4. Thakur J. Key recommendations of high-level expert group report on universal health coverage for India. Indian J Community Med 2011;36:S84-5.

5. Shi L. The impact of primary care: a focused review. Scientifica 2012;2012:e432892:1-22.

6. Dhawan AP, Heetderks WJ, Pavel M, et al. Current and future challenges in point-of-care technologies: a paradigm-shift in affordable global healthcare with personalized and preventive medicine. IEEE J Transl Eng Health Med 2015;3:1-10.

7. Topol EJ. Transforming medicine via digital innovation. Sci Trans/ Med 2010;2:16cm4.

8. Puri RS, Athanassiadis AG, Gill N, et al. Design and preliminary evaluation of a wearable device for mass-screening of sleep apnea. Conf Proc IEEE Eng Med Biol Soc 2016;2016:1870 73.

9. Pamplona VF, Mohan A, Oliveira MM, et al. NETRA: interactive display for estimating refractive errors and focal range. ACM T Graphics 2010;29.

10. Swedish $\mathrm{T}$, Roesch $\mathrm{K}$, Lee $\mathrm{IH}$, et al. EyeSelfie: self directed eye alignment using reciprocal eye box imaging. ACM T Graphics 2015;34.

11. Das A, Swedish T, Wahi A, et al. Mobile phone based minispectrometer for rapid screening of skin cancer. Proc Spie 2015:9482

12. Giller G. Using a smartphone to detect cancer. Sci Am 2014;310:28.

13. Creuzot-Garcher $\mathrm{C}$, Martin-Phipps T, Beynat $\mathrm{J}$, et al. Effectiveness of a mobile diabetic retinopathy screening campaign to encourage diabetics to undergo regular ophthalmic follow-up. Ophthalmic Res 2014;52:206-11.

14. Pirnstill CW, Coté GL. Malaria diagnosis using a mobile phone polarized microscope. Sci Rep 2015;5:13368.

15. Granot $Y$, Ivorra A, Rubinsky B. A new concept for medical imaging centered on cellular phone technology. PLoS One 2008;3:e2075.
16. Jani IV, Quevedo JI, Tobaiwa O, et al. Use of mobile phone technology to improve the quality of point-of-care testing in a lowresource setting. AIDS 2016;30:159-61.

17. Muhlestein JB, Le V, Albert D, et al. Smartphone ECG for evaluation of STEMI: results of the ST LEUIS Pilot Study. J Electrocardiol 2015;48:249-59.

18. Rechmann P, Liou SW, Rechmann BM, et al. Performance of a light fluorescence device for the detection of microbial plaque and gingival inflammation. Clin Oral Investig 2016;20:151-9.

19. Gabel M, Gilad-Bachrach R, Renshaw E, et al. Full body gait analysis with Kinect. Conf Proc IEEE Eng Med Biol Soc 2012;2012:1960-7.

20. Richards JR, Gaylor KA, Pilgrim AJ. Comparison of traditional otoscope to iPhone otoscope in the pediatric ED. Am J Emerg Med 2015;33:1089-92.

21. Eikelboom RH, Mbao MN, Coates HL, et al. Validation of tele-otology to diagnose ear disease in children. Int J Pediatr Otorhinolaryngol 2005;69:739-44.

22. Kopycka-Kedzierawski DT, Bell CH, Billings RJ. Prevalence of dental caries in Early Head Start children as diagnosed using teledentistry. Pediatr Dent 2008;30:329-33.

23. Russo A, Morescalchi F, Costagliola C, et al. A novel device to exploit the smartphone camera for fundus photography. J Ophthalmol 2015;2015:1-5.

24. Baranwal A, Anand A, Singh R, et al. Managing the Earth's Biggest Mass Gathering Event and WASH Conditions: Maha Kumbh Mela (India). PLoS Curr 2015;7:ecurrents.dis.e8b3053f40e774e7e3fdbe1b b50a130d.

25. Nitzan M, Romem A, Koppel R. Pulse oximetry: fundamentals and technology update. Med Devices 2014;7:231-9.

26. Gagnon C, Mathieu J, Desrosiers J. Standardized finger-nose test validity for coordination assessment in an ataxic disorder. Can $\mathrm{J}$ Neurol Sci 2004;31:484-9.

27. Norman KE, Héroux ME. Measures of fine motor skills in people with tremor disorders: appraisal and interpretation. Front Neurol 2013;4:50.

28. Salzman B. Gait and balance disorders in older adults. Am Fam Physician 2010;82:61-8.

29. Spaeth GL, Henderer J, Liu C, et al. The disc damage likelihood scale: reproducibility of a new method of estimating the amount of optic nerve damage caused by glaucoma. Trans Am Ophthalmol Soc 2002;100:181-5. discussion 5-6.

30. Clinical Guidelines on the Identification, Evaluation, and Treatment of Overweight and Obesity in Adults--The Evidence Report. National Institutes of Health. Obes Res 1998;6(Suppl 2):51S-209.

31. Pickering TG, Hall JE, Appel LJ, et al. Recommendations for blood pressure measurement in humans and experimental animals: part 1: blood pressure measurement in humans: a statement for professionals from the Subcommittee of Professional and Public Education of the American Heart Association Council on High Blood Pressure Research. Circulation 2005;111:697-716.

32. Kumar A, Kalra S, Unnikrishnan AG. Metabolic state of the nation: Results of the national family health survey-4. Indian J Endocrinol Metab 2016;20:429-31.

33. Vold ML, Aasebø U, Melbye H. Low FEV1, smoking history, and obesity are factors associated with oxygen saturation decrease in an adult population cohort. Int $J$ Chron Obstruct Pulmon Dis 2014;9:1225-33.

34. DeStefano F, Anda RF, Kahn HS, et al. Dental disease and risk of coronary heart disease and mortality. BMJ 1993;306:688-91.

35. Mendez MV, Scott T, LaMorte W, et al. An association between periodontal disease and peripheral vascular disease. Am J Surg 1998;176:153-7.

36. Jampani ND, Nutalapati R, Dontula BS, et al. Applications of teledentistry: A literature review and update. J Int Soc Prev Community Dent 2011;1:37-44.

37. Veerasamy A, Kirk R, Gage J. Epidemiology of dental caries among adolescents in Tamil Nadu, India. Int Dent J 2016;66:169-77.

38. George R, Ve RS, Vijaya L. Glaucoma in India: estimated burden of disease. J Glaucoma 2010;19:391-7.

39. Mbao MN, Eikelboom RH, Atlas MD, et al. Evaluation of video-otoscopes suitable for tele-otology. Telemed J E Health 2003;9:325-30.

40. Gourie-Devi M. Epidemiology of neurological disorders in India: review of background, prevalence and incidence of epilepsy, stroke, Parkinson's disease and tremors. Neurol India 2014;62:588-98. 